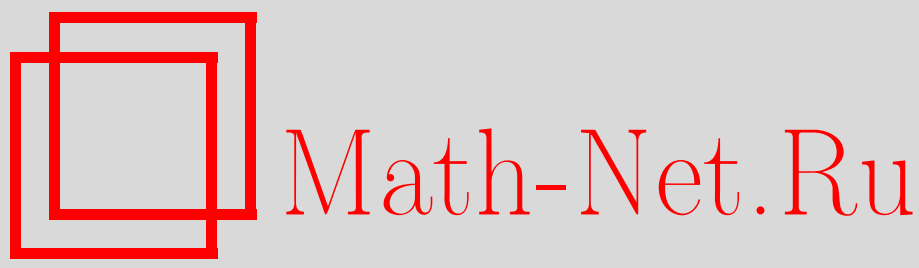

Г. Г. Геворкян, К. А. Навасардян, О рядах Уолша с монотонными коэффициентами, Изв. РАН. Сер. матем., 1999, том 63, выпуск 1, 41-60

DOI: https://doi.org/10.4213/im227

Использование Общероссийского математического портала Math-Net.Ru подразумевает, что вы прочитали и согласны с пользовательским соглашением http://www . mathnet.ru/rus/agreement

Параметры загрузки:

IP : 44.207 .124 .84

26 апреля 2023 г., $16: 40: 24$ 
УДК 517.53

\author{
Г. Г. Геворкян, К. А. Навасардян
}

\title{
О рядах Уолша с монотонными коэффициентами
}

\begin{abstract}
В работе доказано, что если $a_{n} \downarrow 0$ и $\sum_{n=0}^{\infty} a_{n}^{2}=+\infty$, то ряд по системе Уолша $\sum_{n=0}^{\infty} a_{n} W_{n}(x)$ обладает свойством: для любой почти всюду конечной измеримой функции $f(x)$ существуют такие числа $\delta_{n}=0, \pm 1$, что ряд $\sum_{n=0}^{\infty} \delta_{n} a_{n} W_{n}(x)$ почти всюду сходится к $f(x)$. Это утверждение дополняет и усиливает ранее известные результаты об универсальных рядах и о нуль-рядах по системе Уолша.
\end{abstract}

Библиография: 23 наименования.

\section{§ 1. Введение}

Рассматриваются ряды по системе Уолша

$$
\sum_{n=0}^{\infty} a_{n} W_{n}(x)
$$

с монотонными коэффищиентами, т.е.

$$
a_{0} \geqslant a_{1} \geqslant \cdots \geqslant a_{n} \geqslant \cdots, \quad \lim _{n \rightarrow \infty} a_{n}=0 .
$$

Известно (см. [1, с. 149]), что ряд (1) с коэффициентами (2) равномерно сходится на любом интервале вида $(\delta, 1), \delta>0$.

В работе [2] была доказана

Tеорема A. Пусть $\varepsilon_{n} \downarrow 0 u \sum_{n=0}^{\infty} \varepsilon_{n}^{2}=+\infty$. Тогда существует множество $E \subset[0,1], \mu E=0$, которое обладает следующими свойствами:

1) существует ряд $\sum_{n=0}^{\infty} a_{n} W_{n}(x)$, который всюду вне E сходится к нулю, $\left|a_{n}\right| \leqslant \varepsilon_{n}$ u $a_{0} \neq 0$

2) если ряд $\sum_{n=0}^{\infty} b_{n} W_{n}(x)$ всюду вне $E$ сходится $к$ нулю и $b_{n}=o\left(\varepsilon_{n}\right)$, то все коэффициенты $b_{n}$ равны нулю.

Первый пункт этой теоремы дает положительньй ответ на вопрос П. Л. Ульянова [3] о существовании нуль-рядов с допустимо быстро убываюшими коэффициентами.

Сушествование тригонометрических нуль-рядов с допустимо быстро убывающими коэффищиентами независимо было доказано тремя авторами (см. [4]-[6]).

Многомерные аналоги теоремы А доказаны в работах [7], [8].

В настоящей работе мы докажем следующую теорему.

(C) Г.Г. Геворкян, К. А. НАвасардян, 1999 
ТЕОРема 1. Пусть последовательность $\left\{a_{n}\right\}$ удовлетворяет следующим условиям: $a_{n} \downarrow 0$ при $n \rightarrow \infty u \sum_{n=1}^{\infty} a_{n}^{2}=+\infty$. Тогда для любой п.в. конечной измеримой функчии $f(x)$, определенной на $[0,1]$, существует последовательность $\left\{\delta_{n}\right\}, \delta_{n}=0, \pm 1$, такая, что ряд по системе Уолша $\sum_{n=0}^{\infty} \delta_{n} a_{n} W_{n}(x)$ сходится $\kappa f(x)$ п.в. на $[0,1]$. Причем любое конечное число множителей $\delta_{n}$ можно взять произвольным.м.

Очевидно, что условие $a_{n} \downarrow 0$ можно заменить условием $\left|a_{n}\right| \downarrow 0$. Ясно также, что из теоремы 1 следует сушествование нуль-рядов по системе Уолша с допустимо быстро убываюшими коэффициентами.

Пусть $S$ - некоторый класс измеримых функций.

Напомним, что ряд

$$
\sum_{n=0}^{\infty} f_{n}(x)
$$

называется универсальныцми относительно знаков (порядков) в классе $S$, если для любой функции $F(x) \in S$ существует последовательность $\left\{\gamma_{n}\right\}, \gamma_{n}= \pm 1$ $\left(\gamma_{n}=1,0\right)$, для которой ряд $\sum_{n=0}^{\infty} \gamma_{n} f_{n}(x)$ сходится к $F(x)$ п.в.

Ряд (3) назьвается универсальным относительно перестановок в классе $S$, если для любой функции $F(x) \in S$ члены ряда (3) можно переставить так, чтобы вновь полученный ряд сходился к $F(x)$ п.в.

Первые примеры (в разных смыслах) универсальных тригонометрических рядов были построены в работах [9]-[13]. Об исследованиях универсальных ортогональных рядов подробно можно узнать из работ [14], [15].

Здесь отметим один результат Н.Б. Погосяна [16].

ТЕОРемА В. Пусть $\left\{\varepsilon_{n}\right\}_{n=0}^{\infty}-$ произвольная последовательность чисел, удовлетворяющих условиям $\varepsilon_{n} \downarrow 0$ при $n \rightarrow \infty u\left\{\varepsilon_{n}\right\} \notin l_{2}$. Тогда существует функиия $f(x) \in \bigcap_{p<2} L_{p}[0,2 \pi]$ с коэффичиентами Фурье

$a_{n}=\frac{1}{\pi} \int_{0}^{2 \pi} f(x) \cos n x d x, \quad b_{n}=\frac{1}{\pi} \int_{0}^{2 \pi} f(x) \sin n x d x, \quad\left|a_{n}\right|+\left|b_{n}\right| \leqslant \varepsilon_{n}(n \geqslant 0)$,

для которой тригонометрический ряд

$$
\frac{a_{0}}{2}+\sum_{n=1}^{\infty}\left(a_{n} \cos n x+b_{n} \sin n x\right)
$$

является универсальным одновременно относительно знаков, перестановок и подрядов в классе п.в. конечных измеримых функций.

В работе [8] получен неполный аналог теоремы В для кратных рядов по системе Уолша.

Из доказательства теоремы 1 будет видно, что верна следуюшая 
ТЕОРема 2. Пусть последовательность $\left\{a_{n}\right\}$ удовлетворяет следующим условиям: $a_{n} \downarrow 0$ при $n \rightarrow \infty u \sum_{n=1}^{\infty} a_{n}^{2}=+\infty$. Тогда существуют иисла $\gamma_{n}= \pm 1$ такие, что ряд $\sum_{n=0}^{\infty} \gamma_{n} a_{n} W_{n}(x)$ является универсальным относительно подрядов.

Известно (см. [17], [18], а также [1, с. 165]), что ряд (1) с коэффициентами (2) является рядом Фурье-Уолша функции $f(x) \in L_{p}[0,1], 1<p<\infty$, в том и только том случае, если $\sum_{n=1}^{\infty} a_{n}^{p} n^{p-2}<\infty$. Из этого факта и из теоремы 1 следует

Teopema 3. Ряд

$$
\sum_{n=1}^{\infty} \frac{1}{\sqrt{n}} W_{n}(x)
$$

является рядом Фурье-Уолша некоторой функиии из класса $\bigcap_{p<2} L_{p}[0,1], u$ для любой п.в. конечной измеримой функции $f(x)$ существуют числа $\delta_{n}=$ $0, \pm 1$ такие, что ряд $\sum_{n=1}^{\infty} \frac{\delta_{n}}{\sqrt{n}} W_{n}(x)$ n.в. сходится $\kappa f(x)$.

\section{§ 2. Основные леммы}

Напомним, что функции системы Радемахера определяются по формулам

$$
R_{n}(x)=\operatorname{sign}\left(\sin 2^{n} \pi x\right), \quad x \in[0,1], \quad n=1,2, \ldots
$$

Функции системы Уолша выражаются через функции системы Радемахера следующим образом (см., например, $[19$, с. 158$]): W_{0}(x) \equiv 1$, а для $n \geqslant 1$, если $2^{\nu_{1}+\nu_{2}+\cdots+\nu_{p}}\left(\nu_{1}>\nu_{2}>\cdots>\nu_{p}\right)$ есть двоичное представление числа $n$, имеем

$$
W_{n}(x)=\prod_{i=1}^{p} R_{\nu_{i}+1}(x) .
$$

Из определения системы Уолша непосредственно следует, что если $2^{m} \leqslant$ $k<2^{m+1}$ и $n>m$, то

$$
W_{2^{n}}(x) W_{k}(x)=W_{2^{n}+k}(x) .
$$

Пусть $f(x)$ - некоторая функция, а $\sum_{n=0}^{\infty} b_{n} W_{n}(x)$ - ее ряд Фурье по системе Уолша. Через $S^{*}(f, x)$ обозначим мажоранту частичных сумм этого ряда, т.е.

$$
S^{*}(f, x)=\sup _{N}\left|\sum_{n=0}^{N} b_{n} W_{n}(x)\right| .
$$

В работе [7] была доказана следуюшая (см. [7, лемма 3])

ЛЕмма 1. Для любого двоичного интервала $I=\left[\frac{i}{2^{\sigma}}, \frac{i+1}{2^{\sigma}}\right], \quad \sigma \in \mathbb{N}, \quad 0 \leqslant$ $i<2^{\sigma}$, и для любого натурального числа $n>\sigma, n-\sigma$ четное, существует полином по системе Уолша

$$
P(x)=\sum_{k=2^{n}}^{2^{n+1}-1} a_{k} W_{k}(x)
$$


такой, ито:

1) $\left|a_{k}\right|=2^{-\frac{n+\sigma}{2}}=\left(2^{-n} \mu I\right)^{\frac{1}{2}}, 2^{n} \leqslant k<2^{n+1}$;

2) $P(x)=1$, если $x \in E_{1} \subset I, \mu E_{1}=\frac{1}{2} \mu I$;

3) $P(x)=-1$, если $x \in E_{2} \subset I, \mu E_{2}=\frac{1}{2} \mu I$;

4) $P(x)=0$, если $x \notin I$

5) $S^{*}(P, x) \leqslant C$ для всех $x \in[0,1]$;

причем $E_{1}$ и $E_{2}$ являются конечными обгединениями двоичных интервалов, а $C$ - абсолютная постоянная.

С помощью этой леммы и (4) нетрудно доказать следующую лемму.

ЛЕмма 2. Для любого двоичного интервала $I=\left[\frac{i}{2^{\sigma}}, \frac{i+1}{2^{\sigma}}\right], \quad \sigma \in \mathbb{N}, 0 \leqslant i<$ $2^{\sigma}$, и для любых натуральных чисел $m, n u q$, где $n-m-\sigma-1-$ положительное четное число, $0 \leqslant q<2^{m}$, существует полином по системе Уолша $P(x)=\sum_{k=2^{n}}^{2^{n+1}} \delta_{k} W_{k}(x)$ maкой, что:

1) $\delta_{k}= \pm 1$, eслu $2^{n}+q 2^{n-m}+2^{n-m-1} \leqslant k<2^{n}+(q+1) 2^{n-m}$;

2) $\delta_{k}=0$ для остальных $k$;

3) $P(x)=2^{\frac{n-m+\sigma-1}{2}}$, если $x \in E_{1} \subset I, \mu E_{1}=\frac{1}{2} \mu I$;

4) $P(x)=-2^{\frac{n-m+\sigma-1}{2}}$, ecлu $x \in E_{2} \subset I, \mu E_{2}=\frac{1}{2} \mu I$;

5) $P(x)=0$, если $x \notin I$

6) $S^{*}(P, x) \leqslant C \cdot 2^{\frac{n-m+\sigma-1}{2}}$ для всех $x \in[0,1]$;

причем $E_{1}$ и $E_{2}$ являются конечными обвединениями двоичных интервалов, а $C$ - абсолютная постоянная.

ДокАЗАТЕЛЬСТво. Пусть $I=\left[\frac{i}{2^{\sigma}}, \frac{i+1}{2^{\sigma}}\right]-$ двоичный интервал, а $m$ и $n$ - такие натуральные числа, что $n-m-\sigma-1-$ положительное четное число. Тогда согласно лемме 1 существует такой полином

$$
P^{\prime}(x)=\sum_{k=2^{n-m-1}}^{2^{n-m}-1} a_{k} W_{k}(x)
$$

что:

$\left.1^{\prime}\right)\left|a_{k}\right|=2^{-\frac{n-m+\sigma-1}{2}}, 2^{n-m-1} \leqslant k<2^{n-m} ;$

$\left.2^{\prime}\right) P^{\prime}(x)=1$, если $x \in E_{1} \subset I, \mu E_{1}=\frac{1}{2} \mu I$;

$\left.3^{\prime}\right) P^{\prime}(x)=-1$, если $x \in E_{2} \subset I, \mu E_{2}=\frac{1}{2} \mu I$;

$\left.4^{\prime}\right) P^{\prime}(x)=0$, если $x \notin I$;

5) $S^{*}\left(P^{\prime}, x\right) \leqslant C$.

Пусть $0 \leqslant q<2^{m}$ - любое натуральное число и $q=2^{\nu_{1}}+2^{\nu_{2}}+\cdots+2^{\nu_{j}}$. Рассмотрим полином

$$
P(x)=2^{\frac{n-m+\sigma-1}{2}} W_{2^{n-m+\nu_{1}}}(x) \cdots W_{2^{n-m+\nu_{j}}}(x) P^{\prime}(x) W_{2^{n}}(x) .
$$

Ясно, что $P(x)$ удовлетворяет условиям 3$)-6)$ леммы 2. Из (4) и (5) следует, что

$$
P(x)=2^{\frac{n-m+\sigma-1}{2}} \sum_{k=2^{n-m-1}}^{2^{n-m}-1} a_{k} W_{2^{n}+q 2^{n-m}+k}(x)=\sum_{k=2^{n}}^{2^{n+1}-1} \delta_{k} W_{k}(x),
$$


где $\left(\right.$ см. также $\left.\left.1^{\prime}\right)\right)$ числа $\delta_{k}$ удовлетворяют условиям 1) и 2) леммы. Лемма 2 доказана.

Пусть $\left\{\chi_{n}(x)\right\}_{n=0}^{\infty}-$ система Хаара (определение системы Хаара и о связи между системами Хаара и Уолша см., например, [20]). Напомним, что если $2^{n} \leqslant m<$ $2^{n+1}$, то

$$
W_{m}(x)=\frac{1}{2^{\frac{n}{2}}} \sum_{k=2^{n}}^{2^{n+1}-1} \alpha_{k m} \chi_{k}(x), \quad \alpha_{k m}= \pm 1
$$

Пусть $P_{n}(x)=\sum_{k=2^{n}}^{2^{n+1}-1} a_{k} \chi_{k}(x)$ - последовательность полиномов по системе Хаара со следующими свойствами:

а) если $\Delta_{n}=\sup P_{n}(x)=\left\{x: P_{n}(x) \neq 0\right\}$, то полиномы $P_{1}(x), P_{2}(x), \ldots$ $\ldots, P_{n-1}(x)$ принимают постоянные значения на $\Delta_{n}$, и если $\Delta_{n}^{+}=\left\{x: P_{n}(x)>0\right\}$ и $\Delta_{n}^{-}=\left\{x: P_{n}(x)<0\right\}$, то $\left|P_{n}(x)\right|$ - постоянная на множестве $\Delta_{n}^{+} \cup \Delta_{n}^{-}$. Кроме того, для некоторого двоичного интервала $I$ все $\Delta_{n} \subset I$;

b) $\sum_{n=1}^{\infty} P_{n}^{2}(x)=+\infty$ п.в. на интервале $I$;

c) $\sup _{x} S^{*}\left(P_{n}, x\right) \rightarrow 0$ при $n \rightarrow \infty$;

d) $P_{n}(x) \rightarrow 0$ при $n \rightarrow \infty$ для любого $x$.

Тогда справедлива следующая

ЛЕмма 3. Для любых положительных чисел $\varepsilon<1, \delta$ u d существуют полином $P(x)=\sum_{k=1}^{m} P_{n_{k}}(x)$ и множество $E \subset I$ такие, что:

1) $|P(x)-d|<\delta$, если $x \in E, \quad \mu E>(1-\varepsilon) \mu I$;

2) $S^{*}(P, x)<\delta$, если $x \notin I$;

3) $S^{*}(P, x)<C \frac{d}{\varepsilon}+\delta$ для п.в. $x \in I$, где $C$ - абсолютная постоянная.

Для доказательства этой леммы мы пользуемся следующими теоремами.

Теорема С (см. [21], [22] или [19, с. 104]). Для того чтобъ ряд по системе Xaapa

$$
\sum_{n=0}^{\infty} a_{n} \chi_{n}(x)
$$

сходился п.в. на множестве $E \subset[0,1], \mu E>0$, необходимо и достаточно, чтобы для п.в. $x \in E$ била конечна сумма ряда $\sum_{n=1}^{\infty} a_{n}^{2} \chi_{n}^{2}(x)$.

Теорема D (см. [21]-[23] или [19, с. 108]). Если для частичных сумм ряда (7) выполнено соотношение

$$
\limsup _{N \rightarrow \infty} \sum_{n=0}^{N} a_{n} \chi_{n}(x)<+\infty, \quad x \in E, \quad \mu E>0
$$

то ряд (7) сходится п.в. на $E$ (к конечной п.в. функиии). 
ДоКАЗАТЕЛЬСтво ЛЕммы 3. Рассмотрим следуюший ряд:

$$
\sum_{m=1}^{\infty} a_{m} \chi_{m}(x) \equiv \sum_{n=1}^{\infty} P_{n}(x)=\sum_{n=1}^{\infty} \sum_{k=2^{n}}^{2^{n+1}-1} a_{k} \chi_{k}(x) .
$$

Из (8) и свойства b) следует, что $\sum_{m=1}^{\infty} a_{m}^{2} \chi_{m}^{2}(x)=\sum_{n=1}^{\infty} P_{n}^{2}(x)=+\infty$ п.в. на интервале $I$. Поэтому в силу теоремы С ряд (8) расходится п.в. на интервале $I$. Следовательно (см. теорему D),

$$
\limsup _{M \rightarrow \infty} \sum_{m=1}^{M} a_{m} \chi_{m}(x)<+\infty \text { для п.в. } x \in I .
$$

Из (8) и (9) следует, что

$$
\limsup _{N \rightarrow \infty} \sum_{n=1}^{N} P_{n}(x)=+\infty \text { для п.в. } x \in I .
$$

Из свойств с) и d) следует, что существует натуральное число $n_{0}$ такое, что:

$$
\begin{array}{rrr}
S^{*}\left(P_{n}, x\right)<\delta & \text { при } & n>n_{0}, \\
\left|P_{n}(x)\right|<\frac{\delta}{2} & \text { при } & n>n_{0} .
\end{array}
$$

Рассмотрим следуюшую функцию:

$$
\tau(x)=\left\{\begin{array}{l}
+\infty, \text { если } \sup _{N: x \in \Delta_{N}} \sum_{n=n_{0}}^{N} P_{n}(x) \leqslant d, \\
\inf \left\{N: \max _{x \in \Delta_{N+1}} \sum_{n=n_{0}}^{N+1} P_{n}(x)>d\right\} \text { в противном случае. }
\end{array}\right.
$$

Легко видеть, что при $j=1,2, \ldots$ множество $e_{j}=\{x: \tau(x)=j\}$ либо пусто, либо совпадает с $\Delta_{j+1}$. Так как $P_{n}(x)$ - полином по системе Хаара, то из а) получаем, что при $n>j+1$ интервалы $\Delta_{n}$ и $e_{j}$ либо не пересекаются, либо $\Delta_{n} \subset e_{j}$. Положим $\gamma_{n}=0$, если $\Delta_{n} \subset e_{j}$ для некоторого $j$, и $\gamma_{n}=1$ в противном случае. Из выбора чисел $\left\{\gamma_{n}\right\}$ и из (13) следует, что

$$
\sup _{N} \sum_{n=n_{0}}^{N} \gamma_{n} P_{n}(x) \leqslant d, \quad x \in I .
$$

Пусть $x \in I$ и $\lim \sup _{N \rightarrow \infty} \sum_{n=1}^{\infty} P_{n}(x)=+\infty$. Тогда $x \in e_{j}$ для некоторого $j \geqslant n_{0}$, поэтому

$$
\sum_{n=n_{0}}^{\infty} \gamma_{n} P_{n}(x)=\sum_{n=n_{0}}^{j} P_{n}(x), \quad x \in e_{j} .
$$


Но полиномы $P_{n_{0}}(x), P_{n_{0}+1}(x), \ldots, P_{j}(x)$ принимают постоянные значения на интервале $e_{j}=\Delta_{j+1}\left(\right.$ см. свойство а)), а $\sum_{n=n_{0}}^{j+1} P_{n}\left(x^{\prime}\right)>d$ для некоторого $x^{\prime} \in e_{j}$. Следовательно, из (12) и (14) получаем, что для $x \in e_{j}$

$$
\left|\sum_{n=n_{0}}^{\infty} \gamma_{n} P_{n}(x)-d\right|<\frac{\delta}{2}
$$

Учитывая также (10), получаем, что неравенство (15) выполняется для п.в. $x \in I$.

Положим

$$
\tau^{\prime}(x)=\left\{\begin{array}{r}
+\infty, \text { если } \inf _{N: x \in \Delta_{N}}\left(\min _{t \in \Delta_{N}} \sum_{n=n_{0}}^{N} \gamma_{n} P_{n}(t)\right)>-\frac{4 d}{\varepsilon}, \\
\inf \left\{N: \min _{t \in \Delta_{N+1}} \sum_{n=n_{0}}^{N+1} \gamma_{n} P_{n}(x)<-\frac{4 d}{\varepsilon}, x \in \Delta_{N+1}\right\} \\
\text { в противном случае. }
\end{array}\right.
$$

Легко видеть, что при $j \geqslant n_{0}$ множество $e_{j}^{\prime}=\left\{x: \tau^{\prime}(x)=j\right\}$ либо пусто, либо совпадает с $\Delta_{j+1}$. Пусть $\gamma_{n}^{\prime}=0$, если $\Delta_{n} \subset e_{j}^{\prime}$ для некоторого $j$, и $\gamma_{n}^{\prime}=1$ в противном случае. Нетрудно убедиться в том, что:

$$
\begin{aligned}
& \inf _{N} \sum_{n=n_{0}}^{N} \gamma_{n}^{\prime} \gamma_{n} P_{n}(x) \geqslant-\frac{4 d}{\varepsilon} \quad \text { для п.в. } x \in I \text {, } \\
& \sum_{n=n_{0}}^{\infty} \gamma_{n}^{\prime} \gamma_{n} P_{n}(x)=\sum_{n=n_{0}}^{j} \gamma_{n} P_{n}(x), \quad \text { если } x \in e_{j}, \quad j \geqslant n_{0}, \\
& \sum_{n=n_{0}}^{\infty} \gamma_{n}^{\prime} \gamma_{n} P_{n}(x)=\sum_{n=n_{0}}^{\infty} \gamma_{n} P_{n}(x), \quad \text { если } \tau(x)=+\infty \text {. }
\end{aligned}
$$

Из (15) и (19) следует, что

$$
\left|\sum_{n=n_{0}}^{\infty} \gamma_{n}^{\prime} \gamma_{n} P_{n}(x)-d\right|<\frac{\delta}{2} \quad \text { для п.в. } x \in\left\{x: \tau^{\prime}(x)=+\infty\right\} .
$$

Докажем, что

$$
\mu\left\{x: \tau^{\prime}(x)=+\infty\right\}>\left(1-\frac{\varepsilon}{2}\right) \mu I .
$$

Действительно, из (16) имеем

$$
\mu\left\{x: \tau^{\prime}(x)=+\infty\right\}=\mu I-\mu\left\{x: \tau^{\prime}(x)<+\infty\right\}=\mu I-\sum_{j=n_{0}}^{\infty} \mu\left\{x: \tau^{\prime}(x)=j\right\}
$$


и на множестве $e_{j}^{\prime}=\left\{x: \tau^{\prime}(x)=j\right\}=\Delta_{j+1}\left(\right.$ если $\left.e_{j}^{\prime} \neq \varnothing\right)$ сумма $\sum_{n=n_{0}}^{j} \gamma_{n} P_{n}(x)$ постоянная (см. свойство а)). Следовательно, на одном из множеств $\Delta_{j+1}^{+}$или $\Delta_{j+1}^{-}$выполняется неравенство

$$
\sum_{n=n_{0}}^{j+1} \gamma_{n} P_{n}(x)<-\frac{4 d}{\varepsilon}
$$

Поэтому

$$
\mu e_{j}^{\prime} \leqslant 2 \mu\left\{x \in \Delta_{j+1}: \sum_{n=n_{0}}^{j+1} \gamma_{n} P_{n}(x)<-\frac{4 d}{\varepsilon}\right\} .
$$

Рассмотрим функцию

$$
S(x)= \begin{cases}\sum_{n=n_{0}}^{j+1} \gamma_{n} P_{n}(x), & \text { если } x \in e_{j}^{\prime}, \\ \sum_{n=n_{0}}^{\infty} \gamma_{n} P_{n}(x), & \text { если } \tau^{\prime}(x)=+\infty\end{cases}
$$

Так как множества $e_{j}^{\prime}, j \geqslant n_{0}$, не пересекаются, $\int_{0}^{1} P_{n}(x) d x=0$ для всех $n \geqslant n_{0}$ $\left(P_{n}(x)\right.$ - полином по системе Хаара) и

$$
\sup _{N}\left|\sum_{n=n_{0}}^{N} \gamma_{n}^{\prime} \gamma_{n} P_{n}(x)\right| \leqslant d+\frac{4 d}{\varepsilon} \text { для п.в. } x \in I
$$

(см. (14) и (17)), то из (18), (19) и (23) следует, что

$$
\begin{aligned}
\int_{0}^{1} S(x) d x & =\int_{0}^{1} \sum_{n=n_{0}}^{\infty} \gamma_{n}^{\prime} \gamma_{n} P_{n}(x) d x+\int_{0}^{1} \sum_{\substack{n=n_{0} \\
e_{n}^{\prime} \neq 0}}^{\infty} \gamma_{n+1} P_{n+1}(x) \\
& =\sum_{n=n_{0}}^{\infty} \gamma_{n}^{\prime} \gamma_{n} \int_{0}^{1} P_{n}(x) d x+\sum_{\substack{n=n_{0} \\
e_{n}^{\prime} \neq 0}}^{\infty} \gamma_{n+1} \int_{0}^{1} P_{n+1}(x) d x=0 .
\end{aligned}
$$

Учитывая также (14), получаем

$$
0=\int_{0}^{1} S(x) d x \leqslant d \mu I-\frac{4 d}{\varepsilon} \mu\left\{x: S(x)<-\frac{4 d}{\varepsilon}\right\}
$$

откуда

$$
\mu\left\{x: S(x)<-\frac{4 d}{\varepsilon}\right\}<\frac{\varepsilon}{4} \mu I .
$$


Поэтому (см. также (21)-(23))

$$
\begin{aligned}
\mu\left\{x: \tau^{\prime}(x)=+\infty\right\} & \geqslant \mu I-2 \sum_{j=n_{0}}^{\infty} \mu\left\{x \in e_{j}^{\prime}: \sum_{n=n_{0}}^{j+1} \gamma_{n} P_{n}(x)<-\frac{4 d}{\varepsilon}\right\} \\
& =\mu I-2 \mu\left\{x \in I: S(x)<-\frac{4 d}{\varepsilon}\right\}>\left(1-\frac{\varepsilon}{2}\right) \mu I .
\end{aligned}
$$

Из (20) и (25) следует, что существуют натуральное число $m>n_{0}$ и множество $E$ такие, что

$$
\begin{array}{ll}
E \subset\left\{x: \tau^{\prime}(x)=+\infty\right\}, & \mu E>(1-\varepsilon) \mu I, \\
\left|\sum_{n=n_{0}}^{m} \gamma_{n}^{\prime} \gamma_{n} P_{n}(x)-d\right|<\delta, & x \in E .
\end{array}
$$

Положив $P(x)=\sum_{n=n_{0}}^{m} \gamma_{n}^{\prime} \gamma_{n} P_{n}(x)$, нетрудно убедиться в том, что (см. (11) и свойство а))

$$
S^{*}(P, x)<\delta, \text { если } x \notin I
$$

и (см. (11) и (24))

$$
S^{*}(P, x)<d\left(1+\frac{4}{\varepsilon}\right)+\delta<\frac{5 d}{\varepsilon}+\delta \quad \text { для п.в. } x \in I .
$$

Тем самым лемма 3 доказана.

\section{§ 3. Главная лемма}

ЛЕмма 4. Пусть $a_{n} \downarrow 0 u \sum_{n \geqslant 0} a_{n}^{2}=+\infty$. Тогда для любого двоичного интервала $I=\left[\frac{\eta}{2^{\sigma}}, \frac{\eta+1}{2^{\sigma}}\right], \quad \sigma \in \mathbb{N}, \quad 0 \leqslant \eta<2^{\sigma}$, и для любых положсительных чисел $\varepsilon<1, \delta<1, d, M(M \in \mathbb{N})$ существуют полином по системе Уолша $P(x)=\sum_{n=M}^{N} \delta_{n} a_{n} W_{n}(x)$ и множсество $E \in[0,1]$ такие, что:

1) $\delta_{n}=0, \pm 1$ для $M \leqslant n \leqslant N$;

2) $\left|P(x)-d \chi_{I}(x)\right|<\delta$, ecлu $x \notin E$;

3) $\mu E<\varepsilon \mu I$;

4) $S^{*}(P, x)<\delta$, если $x \notin I \cup E$;

5) $S^{*}(P, x)<C \frac{d}{\varepsilon}+\delta$, если $x \in I \backslash E$;

где $\chi_{I}(x)$ - характеристическая функция интервала $I$, а $C$ - абсолютная постоянная. 
ДоКАЗАТЕЛЬСТВО. Пусть $a_{n} \downarrow 0$ и $\sum_{n \geqslant 0} a_{n}^{2}=+\infty$. Тогда

$$
\sum_{n \geqslant 0} 2^{n} a_{2^{n}}^{2}=+\infty
$$

Обозначим

$$
G=\left\{k \in \mathbb{N}: a_{2^{k}}<2 a_{2^{k+1}}\right\} .
$$

Пусть $m \in G, m<n$ и $k \notin G$ при $m<k<n$; тогда

$$
\sum_{k=m+1}^{n} 2^{k} a_{2^{k}}^{2} \leqslant \sum_{k=m+1}^{n} 2^{k}\left(\frac{a_{2^{m+1}}}{2^{k-m-1}}\right)^{2}=2^{m} a_{2^{m+1}}^{2} \sum_{k=m+1}^{n} \frac{1}{2^{k-m-2}}<4 \cdot 2^{m} a_{2^{m}}^{2} .
$$

Отсюда следует, что

$$
\sum_{k=0}^{\infty} 2^{k} a_{2^{k}}^{2} \leqslant 4 a_{1}^{2}+4 \sum_{m \in G} 2^{m} a_{2^{m}}^{2}
$$

Из последнего неравенства с учетом (26) получаем

$$
\sum_{k \in G} 2^{k} a_{2^{k}}^{2}=+\infty
$$

Не ограничивая обшности, мы можем считать, что если $k, k^{\prime} \in G$, то $\left(k-k^{\prime}\right)-$ четное число. Действительно, за $G$ можно взять одно из следующих множеств: $G_{1}=\{k \in G: k$ четное $\}$ и $G_{2}=\{k \in G: k$ нечетное $\}$. Очевидно, что для одного из них выполняется (28).

Пусть $\gamma$-некоторое число, удовлетворяющее условию

$$
\gamma^{2}<\left((d+1)^{2}+\frac{4(C d+1)^{2}}{\varepsilon}\right)^{-1} \cdot \frac{\delta^{2} \varepsilon}{16} \mu I
$$

где $C$ - постоянная из леммы 3.

Возьмем натуральное число $m$ настолько большим, чтобы выполнялось неравенство

$$
(1+\gamma)^{2^{m}}>2
$$

и рассмотрим следуюшие отношения:

$$
\frac{a_{2^{n}+q 2^{n-m}}}{a_{2^{n}+(q+1) 2^{n-m}}}, \quad n \in G, \quad n>m, \quad q=1,2, \ldots, 2^{m} .
$$

Из (27) и (30) следует, что для каждого $n \in G, n>m$, существует такое число $q_{n}$, что

$$
\frac{a_{2^{n}+q_{n} 2^{n-m}}}{a_{2^{n}+\left(q_{n}+1\right) 2^{n-m}}}<1+\gamma, \quad n \in G, \quad n>m .
$$

Обозначим (в дальнейшем будем считать, что если $n \in G$, то $n>m$ )

$$
\alpha_{n}=a_{2^{n}+\left(q_{n}+1\right) 2^{n-m}}, \quad n \in G .
$$


Очевидно, что

$$
\frac{a_{k}}{\alpha_{n}}-1<\gamma, \text { если } 2^{n}+q_{n} 2^{n-m} \leqslant k \leqslant 2^{n}+\left(q_{n}+1\right) 2^{n-m}
$$

Из (27) и (32) с учетом монотонности последовательности $\left\{a_{n}\right\}$ следует, что

$$
\sum_{n \in G} 2^{n} \alpha_{n}^{2} \geqslant \sum_{n \in G} 2^{n} \alpha_{2^{n+1}}^{2} \geqslant 2^{-2} \sum_{n \in G} 2^{n} a_{2^{n}}^{2}
$$

Поэтому (см. также (28))

$$
\sum_{n \in G} 2^{n} \alpha_{n}^{2}=+\infty
$$

Обозначим $G^{\prime}=\left\{n \in G: 2^{n} \alpha_{n}^{2}>2^{-n / 2}, n>m\right\}$. Очевидно, что

$$
\sum_{n \in G \backslash G^{\prime}} 2^{n} \alpha_{n}^{2} \leqslant \sum_{n \in G \backslash G^{\prime}} 2^{-n / 2}<+\infty .
$$

Следовательно (см. также (34)),

$$
\sum_{n \in G^{\prime}} 2^{n} \alpha_{n}^{2}=+\infty
$$

Сушествуют натуральные числа $p_{n}$ такие, что

$$
\begin{aligned}
& n-m-p_{n}-\text { четное число, } n \in G^{\prime} \text {, } \\
& \sum_{n \in G^{\prime}} 2^{n-m-p_{n}} \alpha_{n}^{2}=+\infty \\
& \lim _{\substack{n \rightarrow \infty \\
n \in G^{\prime}}} 2^{n-m-p_{n}} \alpha_{n}^{2}=0, \\
& \lim _{\substack{n \rightarrow \infty \\
n \in G^{\prime}}} 2^{n-m-p_{n}} \alpha_{n}=+\infty .
\end{aligned}
$$

В дальнейшем через $n^{\prime}$ будем обозначать число $n-m-p_{n}$. Пусть $I=\left[\frac{\eta}{2^{\sigma}}, \frac{\eta+1}{2^{\sigma}}\right]-$ двоичный интервал, а $M$ - некоторое натуральное число. Из (37)-(39) следует, что существует конечное подмножество $B_{1}^{\prime}$ множества $G^{\prime}$ такое, что

$$
\begin{gathered}
\min \left\{B_{1}^{\prime}\right\} \geqslant \log _{2} M, \\
2^{n^{\prime}} \alpha_{n}>4, \quad \text { если } n \in B_{1}^{\prime}, \\
2^{n^{\prime}} \alpha_{n}^{2}<2^{-\sigma}, \quad \text { если } n \in B_{1}^{\prime}, \\
\sum_{n \in B_{1}^{\prime}} 2^{n^{\prime}} \alpha_{n}^{2}>2^{-\sigma+1} .
\end{gathered}
$$


Для каждого $n \in B_{1}^{\prime}$ сушествует нечетное число $\sigma_{n} \geqslant \sigma$ такое, что (см. (42))

$$
2^{-\sigma_{n}-1}<2^{n^{\prime}} \alpha_{n}^{2} \leqslant 2^{-\sigma_{n}+1} .
$$

Из (41) и (44) следует, что

$$
2^{n^{\prime}-\sigma_{n}} \geqslant 2^{n^{\prime}} \cdot 2^{n^{\prime}-1} \cdot \alpha_{n}^{2}=\frac{1}{2}\left(2^{n^{\prime}} \alpha_{n}\right)^{2}>8, \quad n \in B_{1}^{\prime},
$$

т.е. (см. также (36))

$$
n^{\prime}-\sigma_{n}-1>0, n^{\prime}-\sigma_{n}-1 \text { - четное число, } n \in B_{1}^{\prime} \text {. }
$$

А из (43) и (44) получаем, что

$$
\sum_{n \in B_{1}^{\prime}} 2^{-\sigma_{n}} \geqslant \frac{1}{2} \sum_{n \in B_{1}^{\prime}} 2^{n^{\prime}} \alpha_{n}^{2}>2^{-\sigma}
$$

Следовательно (напомним, что $\sigma_{n} \geqslant \sigma$ ), существует подмножество $B_{1}$ множества $B_{1}^{\prime}$ такое, что

$$
\sum_{n \in B_{1}} 2^{-\sigma_{n}}=2^{-\sigma}
$$

Интервал $I$ представим в виде объединения двоичных интервалов $I_{n}, n \in B_{1}$, $\mu I_{n}=2^{-\sigma_{n}}$. В силу леммы 2 (см. также (45)) для каждого интервала $I_{n}, n \in B_{1}$, и чисел $m+p_{n}, n$ и $q_{n} 2^{p_{n}}$ существует полином по системе Уолша

$$
P_{n}(x)=\sum_{k=2^{n}}^{2^{n+1}-1} \alpha_{n} \delta_{k} W_{k}(x)
$$

такой, что:

$\left.1^{\prime}\right) \delta_{k}= \pm 1$, если $2^{n}+q_{n} 2^{n-m}+2^{n-m-p_{n}-1} \leqslant k<2^{n}+q_{n} 2^{n-m}+2^{n-m-p_{n}}$;

$\left.2^{\prime}\right) \delta_{k}=0$ для остальных $k$;

$\left.3^{\prime}\right) P_{n}(x)=2^{\frac{n^{\prime}+\sigma_{n}-1}{2}} \alpha_{n}$, если $x \in E_{n}^{\prime}, \mu E_{n}^{\prime}=\frac{1}{2} \mu I_{n}$;

4') $P_{n}(x)=-2^{\frac{n^{\prime}+\sigma_{n}-1}{2}} \alpha_{n}$, если $x \in E_{n}^{\prime \prime}, \mu E_{n}^{\prime \prime}=\frac{1}{2} \mu I_{n}$;

5') $P_{n}(x)=0$, если $x \notin I_{n}$;

причем $E_{n}^{\prime}$ и $E_{n}^{\prime \prime}, n \in B_{1}$, являются конечными объединениями двоичных интервалов. Из (44), $\left.3^{\prime}\right)$ и $4^{\prime}$ ) следует, что

$$
2^{-1} \leqslant\left|P_{n}(x)\right|=2^{\frac{n^{\prime}+\sigma_{n}-1}{2}} \alpha_{n} \leqslant 1, \quad \text { если } x \in E_{n}^{\prime} \cup E_{n}^{\prime \prime}, \quad n \in B_{1} .
$$

Допустим, что множества $B_{1}, B_{2}, \ldots, B_{j-1}$ и полиномы $P_{n}(x), n \in \bigcup_{i=1}^{j-1} B_{i}$, уже построены, $\lambda_{j-1}=\max B_{j-1}+1$ и полином $P_{n}(x), n \in \bigcup_{i=1}^{j-1} B_{i}$, постоянный на каждом двоичном интервале с длиной $2^{-\lambda_{j-1}}$. 
Из (37)-(39) следует, что существует конечное подмножество $B_{j}^{\prime}$ множества $G^{\prime}$ такое, что:

$$
\begin{gathered}
\min B_{j}^{\prime} \geqslant \lambda_{j-1}, \\
2^{n^{\prime}} \alpha_{n}>\frac{4}{\sqrt{j}}, \text { если } n \in B_{j}^{\prime}, \\
2^{n^{\prime}} \alpha_{n}^{2} \leqslant \frac{1}{j} 2^{-\lambda_{j-1}}, \text { если } n \in B_{j}^{\prime}, \\
\sum_{n \in B_{j}^{\prime}} 2^{n^{\prime}} \alpha_{n}^{2}>2^{-\sigma+1} \cdot \frac{1}{j} .
\end{gathered}
$$

Для каждого $n \in B_{j}^{\prime}$ существует нечетное число $\sigma_{n} \geqslant \lambda_{j-1}$ такое, что (см. (48))

$$
\frac{1}{j} 2^{-\sigma_{n}-1}<2^{n^{\prime}} \alpha_{n}^{2} \leqslant \frac{1}{j} 2^{-\sigma_{n}+1} .
$$

Из (47) и (50) следует, что

$$
2^{n^{\prime}-\sigma_{n}} \geqslant 2^{n^{\prime}} j 2^{n^{\prime}-1} \alpha_{n}^{2}=\frac{j}{2}\left(2^{n^{\prime}} \alpha_{n}\right)^{2}>8, \quad n \in B_{j}^{\prime},
$$

т.е. (см. также (36)) $n^{\prime}-\sigma_{n}-1>0, n^{\prime}-\sigma_{n}-1$ - четное число, $n \in B_{j}^{\prime}$.

Из (49) и (50) получаем, что

$$
\sum_{n \in B_{j}^{\prime}} 2^{-\sigma_{n}} \geqslant \frac{j}{2} \sum_{n \in B_{j}^{\prime}} 2^{n^{\prime}} \alpha_{n}^{2}>2^{-\sigma} .
$$

Следовательно (напомним, что $\sigma_{n} \geqslant \lambda_{j-1}>\sigma$ ), существует подмножество $B_{j}$ множества $B_{j}^{\prime}$ такое, что

$$
\sum_{n \in B_{j}} 2^{-\sigma_{n}}=2^{-\sigma}
$$

Поэтому интервал $I$ можно представить в виде объединения двоичных интервалов $I_{n}, n \in B_{j}, \mu I_{n}=2^{-\sigma_{n}}$. Очевидно, что полином $P_{k}(x), k \in \bigcup_{i=1}^{j-1} B_{i}$, принимает постоянное значение на каждом интервале $I_{n}, n \in B_{j}$. В силу леммы 2 для каждого интервала $I_{n}, n \in B_{j}$, и чисел $m+p_{n}, n$ и $q_{n} 2^{p_{n}}$ существует полином по системе Уолша

$$
P_{n}(x)=\sum_{k=2^{n}}^{2^{n+1}-1} \alpha_{n} \delta_{k} W_{k}(x)
$$

такой, что:

$\left.1^{\prime \prime}\right) \delta_{k}= \pm 1$, если $2^{n}+q_{n} 2^{n-m}+2^{n^{\prime}-1} \leqslant k<2^{n}+q_{n} 2^{n-m}+2^{n^{\prime}}$;

$\left.2^{\prime \prime}\right) \delta_{k}=0$ для остальных $k$;

$\left.3^{\prime \prime}\right) P_{n}(x)=2^{\frac{n^{\prime}+\sigma_{n}-1}{2}} \alpha_{n}$, если $x \in E_{n}^{\prime} \subset I_{n}, \mu E_{n}^{\prime}=\frac{1}{2} \mu I_{n}$;

$\left.4^{\prime \prime}\right) P_{n}(x)=-2^{\frac{n^{\prime}+\sigma_{n}-1}{2}} \alpha_{n}$, если $x \in E_{n}^{\prime \prime} \subset I_{n}, \mu E_{n}^{\prime \prime}=\frac{1}{2} \mu I_{n}$; 
$\left.5^{\prime \prime}\right) P_{n}(x)=0$, если $x \notin I_{n}$;

$\left.6^{\prime \prime}\right) S^{*}\left(P_{n}, x\right) \leqslant C_{1} \cdot 2^{\frac{n^{\prime}+\sigma_{n}-1}{2}} \alpha_{n}$ для всех $x \in[0,1]$;

причем $E_{n}^{\prime}$ и $E_{n}^{\prime \prime}$ являются конечными объединениями двоичных интервалов с длиной $2^{-n-1}$, а $C_{1}$ - постоянная.

Из $\left.\left.\left.3^{\prime \prime}\right), 4^{\prime \prime}\right), 6^{\prime \prime}\right)$ и (50) следует, что

$$
\frac{2^{-1}}{\sqrt{j}} \leqslant\left|P_{n}(x)\right|=2^{\frac{n^{\prime}+\sigma_{n}-1}{2}} \alpha_{n} \leqslant \frac{1}{\sqrt{j}}, \quad \text { если } x \in E_{n}^{\prime} \cup E_{n}^{\prime \prime}, \quad n \in B_{j},
$$

и

$$
S^{*}\left(P_{n}, x\right) \leqslant \frac{C_{1}}{\sqrt{j}} \quad \text { для всех } x \in[0,1] .
$$

Поэтому (см. также $\left.\left.5^{\prime \prime}\right)\right)$

$$
\sum_{n \in B_{j}}\left(P_{n}(x)\right)^{2}>\frac{1}{4 j} \text { для п.в. } x \in I .
$$

Нетрудно убедиться в том, что для полиномов $P_{n}(x), n \in B_{j}, j=1,2, \ldots$, выполняются условия а)-d). Поэтому согласно лемме 3 существует полином

$$
P^{\prime}(x)=\sum_{k=1}^{m} P_{n_{k}}(x), \quad n_{k} \in \bigcup_{j=1}^{\infty} B_{j}
$$

удовлетворяюший следуюшим условиям:

$$
\begin{aligned}
P^{\prime}(x) & =0, \text { если } x \notin I, \\
\mu\{x & \left.\in I:\left|P^{\prime}(x)-d\right|>\frac{\delta}{2}\right\}<\frac{\varepsilon}{4} \mu I, \\
S^{*}\left(P^{\prime}, x\right) & <\frac{\delta}{2}, \text { если } x \notin I, \\
S^{*}\left(P^{\prime}, x\right) & <C \frac{4 d}{\varepsilon}+\frac{\delta}{2} \text { для п.в. } x \in I,
\end{aligned}
$$

где $C$ - абсолютная постоянная из леммы 3.

Из (52), (53) и (55) следует, что (см. также (29))

$$
\left\|P^{\prime}\right\|_{2}^{2} \leqslant\left(d+\frac{\delta}{2}\right)^{2} \mu I+\left(\frac{4 C d}{\varepsilon}+\frac{\delta}{2}\right)^{2} \cdot \frac{\varepsilon}{4} \leqslant(d+1)^{2}+\frac{4(C d+1)^{2}}{\varepsilon}<\frac{\varepsilon \delta^{2}}{16 \gamma^{2}} \mu I .
$$

Для каждого полинома $P_{n}(x), n \in B_{j}, j=1,2, \ldots$ (см. (51)), обозначим

$$
P_{n}^{\prime}(x)=\sum_{k=2^{n}}^{2^{n+1}-1} a_{k} \delta_{k} W_{k}(x)
$$


и рассмотрим полином

$$
P(x)=\sum_{k=M}^{N} \delta_{k} a_{k} W_{k}(x) \equiv \sum_{k=1}^{m} P_{n_{k}}^{\prime}(x) .
$$

Из (33), (51), (57), $\left.1^{\prime \prime}\right)$ и $\left.2^{\prime \prime}\right)$ следует, что

$$
\begin{aligned}
\left\|P-P^{\prime}\right\|_{2}^{2} & =\sum_{k=1}^{m} \sum_{i=2^{n_{k}}}^{2^{n_{k}+1}-1} \delta_{i}^{2}\left(a_{i}-\alpha_{n_{k}}\right)^{2} \\
& =\sum_{k=1}^{m} \sum_{i=2^{n_{k}}}^{2^{n_{k}+1}-1} \delta_{i}^{2} \alpha_{n_{k}}^{2}\left(\frac{a_{i}}{\alpha_{n_{k}}}-1\right)^{2}<\gamma^{2} \sum_{k=1}^{m}\left\|P_{n_{k}}\right\|_{2}^{2}=\gamma^{2}\left\|P^{\prime}\right\|_{2}^{2} .
\end{aligned}
$$

Поэтому с учетом (56) получаем

$$
\left\|P-P^{\prime}\right\|_{2}^{2} \leqslant \frac{\varepsilon \delta^{2}}{16} \mu I
$$

Следовательно,

$$
\mu\left\{x \in[0,1]:\left|P(x)-P^{\prime}(x)\right|>\frac{\delta}{2}\right\} \leqslant \frac{4\left\|P-P^{\prime}\right\|_{2}^{2}}{\delta^{2}}<\frac{\varepsilon}{4} \mu I .
$$

Из (52), (53) и (59) получаем

$$
\mu\left\{x \in[0,1]:\left|P(x)-d \chi_{I}(x)\right|>\delta\right\}<\frac{\varepsilon}{2} \mu I .
$$

Для мажоранты частичных сумм полинома $P(x)$ имеем

$$
S^{*}(P, x) \leqslant S^{*}\left(P^{\prime}, x\right)+S^{*}\left(P-P^{\prime}, x\right) .
$$

Так как система Уолша имеет слабый тип $(2,2)$ (см., например, [1, с. 189]), то из (58) следует, что

$$
\mu\left\{x: S^{*}\left(P-P^{\prime}, x\right)>\frac{\delta}{2}\right\} \leqslant \frac{4 C^{\prime}}{\delta^{2}}\left\|P-P^{\prime}\right\|_{2}^{2}<C^{\prime} \frac{\varepsilon}{2} \mu I,
$$

где $C^{\prime}$ - абсолютная постоянная.

Обозначим

$$
E=\left\{x:\left|P(x)-d \chi_{I}(x)\right|>\delta\right\} \cup\left\{x: S^{*}\left(P-P^{\prime}, x\right)>\frac{\delta}{2}\right\} .
$$

Из (60), (62) и (63) следует, что $\mu E<\left(C^{\prime}+1\right) \varepsilon \mu I$. А из (54), (55), (61) и (63) получаем, что

$$
\begin{aligned}
& S^{*}(P, x)<\delta, \quad \text { если } x \notin E \cup I, \\
& S^{*}(P, x)<\frac{4 C d}{\varepsilon}+\delta, \text { если } x \in I \backslash E .
\end{aligned}
$$

Лемма 4 доказана. 


\section{§4. Доказательство теорем}

Рассмотрим множество $\{(\Delta, d, \lambda)\}$, зависящее от трех параметров, где $\Delta$ пробегает все двоичные интервалы, $d$ пробегает множество всех рациональных чисел, а $\lambda$ - множество положительных рациональных чисел. Пронумеровав это множество, мы можем представить его в виде последовательности

$$
\left(\Delta_{1}, d_{1}, \lambda_{1}\right),\left(\Delta_{2}, d_{2}, \lambda_{2}\right), \ldots,\left(\Delta_{m}, d_{m}, \lambda_{m}\right), \ldots
$$

В этой последовательности элементы отличны друг от друга, но параметры, стоящие в разных элементах в одинаковых местах, могут совпадать.

Обозначим

$$
g_{m}(x)=d_{m} \chi_{\Delta_{m}}(x)=\left\{\begin{array}{lll}
d_{m}, & \text { если } & x \in \Delta_{m}, \\
0, & \text { если } & x \notin \Delta_{m}
\end{array}\right.
$$

Возьмем последовательность положительных чисел $\left\{\eta_{m}\right\}_{m=1}^{\infty}$, где

$$
1>\eta_{1}>\eta_{2}>\cdots, \quad \sum_{m=1}^{\infty} \eta_{m}<+\infty .
$$

В силу леммы 4 для каждого $m=1,2, \ldots$ существуют полином

$$
P_{m}(x)=\sum_{n=M_{m}+1}^{M_{m+1}} \delta_{n} a_{n} W_{n}(x),
$$

множество $E_{m} \subset[0,1]$ и абсолютная постоянная $C$ такие, что:

А) $\delta_{n}=0, \pm 1$ для всех $n$;

В) $\left|P_{m}(x)-g_{m}(x)\right|<\eta_{m}$, если $x \notin E_{m}$;

C) $\mu E_{m}<\lambda_{m} \mu \Delta_{m}$;

D) $S^{*}\left(P_{m}, x\right)<C \frac{\left|d_{m}\right|}{\lambda_{m}}+\eta_{m}$, если $x \in \Delta_{m} \backslash E_{m}$;

Е) $S^{*}\left(P_{m}, x\right)<\eta_{m}$, если $x \notin I \cup E_{m}$.

Докажем, что для любой п.в. конечной измеримой функции $f(x)$ сушествует подпоследовательность $\left\{P_{n_{k}}(x)\right\}$, для которой

$$
\sum_{k=1}^{\infty} P_{n_{k}}(x)=f(x) \text { для п.в. } x \in[0,1] .
$$

Тем самым теорема будет доказана.

Для этого сначала докажем следующую лемму.

ЛЕмма 5. Пусть $\varphi(x)$ - п.в. конечная измеримая функиия, определенная на $[0,1] ;$ далее, пусть $\varepsilon>0, \delta>0$ - произвольные числа, а $m$ - произвольное натуральное число. Тогда существуют натуральные числа $m_{1}, m_{2}, \ldots, m_{j} u$ множество $Е$, удовлетворяющие следующим условиям:

a) $m<m_{1}<m_{2}<\cdots<m_{j}$;

b) $E \subset[0,1], \mu E>1-2 \varepsilon$;

c) $\left|\sum_{i=1}^{j} P_{m_{i}}(x)-\varphi(x)\right|<\delta$, ecлu $x \in E$;

d) $S^{*}\left(\sum_{i=1}^{j} P_{m_{i}}, x\right) \leqslant C \frac{|\varphi(x)|}{\varepsilon}+\varepsilon$ для всех $x \in E$, где $C$ - абсолютная постоянная. 
ДокАЗАТЕЛЬСТво. Легко видеть, что сушествуют конечное разбиение интервала $[0,1]$ на попарно непересекаюшиеся двоичные интервалы $\Delta_{1}^{\prime}, \Delta_{2}^{\prime}, \ldots, \Delta_{j}^{\prime}$, paциональные числа $d_{1}^{\prime}, d_{2}^{\prime}, \ldots, d_{j}^{\prime}$ и множество $E_{0}$ такие, что

$$
\begin{aligned}
E_{0} & \subset[0,1], \quad \mu E_{0}>1-\frac{\varepsilon}{2}, \\
\left|\varphi(x)-d_{i}^{\prime}\right| & <\min \left\{\frac{\delta}{2}, \frac{\varepsilon^{2}}{C}\right\} \text { при } x \in \Delta_{i}^{\prime} \cap E_{0}, \quad i=1,2, \ldots, j,
\end{aligned}
$$

где $C$ - постоянная из D).

Для фиксированного $i, 1 \leqslant i \leqslant j$, в последовательности (64) существует подпоследовательность вида

$$
\left(\Delta_{i}^{\prime}, d_{i}^{\prime}, \lambda_{k_{1}}\right),\left(\Delta_{i}^{\prime}, d_{i}^{\prime}, \lambda_{k_{2}}\right), \ldots,\left(\Delta_{i}^{\prime}, d_{i}^{\prime}, \lambda_{k_{n}}\right), \ldots
$$

где

$$
\lambda_{k_{n}} \geqslant \varepsilon, \quad n=1,2, \ldots, \quad \lim _{n \rightarrow \infty} \lambda_{k_{n}}=\varepsilon .
$$

Пусть $m_{0}$ выбрано настолько большим, чтобы (см. (66))

$$
\sum_{m=m_{0}}^{\infty} \eta_{m}<\min \left\{\varepsilon, \frac{\delta}{2}\right\}
$$

Применяя условия В) $-\mathrm{E}$ ) для $m=k_{n}$ с достаточно большим индексом $n$ и учитывая (68)-(70), легко видеть, что для номера $m_{i}=k_{n}>m_{i-1}$ выполняются условия:

$$
\left|P_{m_{i}}(x)-d_{i}^{\prime}\right|<\eta_{m_{i}}, \text { если } x \in \Delta_{i}^{\prime} \backslash E_{m_{i}},
$$

где

$$
\begin{aligned}
E_{m_{i}} & \subset[0,1], \quad \mu E_{m_{i}}<\lambda_{k_{m}} \mu \Delta_{i}^{\prime}<\varepsilon \mu \Delta_{i}^{\prime}+\frac{\varepsilon}{2 j}, \\
\left|P_{m_{i}}(x)\right| & <\eta_{m_{i}}, \quad \text { если } x \notin \Delta_{i}^{\prime} \cup E_{m_{i}}, \\
S^{*}\left(P_{m_{i}}, x\right) & <\eta_{m_{i}}, \quad \text { если } x \notin \Delta_{i}^{\prime} \cup E_{m_{i}}, \\
S^{*}\left(P_{m_{i}}, x\right) & <C \frac{\left|d_{i}^{\prime}\right|}{\lambda_{k_{n}}}+\eta_{m_{i}}<C \frac{|\varphi(x)|+\varepsilon^{2} / C}{\varepsilon}+\eta_{m_{i}} \\
& <C \frac{|\varphi(x)|}{\varepsilon}+2 \varepsilon, \quad x \in\left(\Delta_{i}^{\prime} \backslash E_{m_{i}}\right) \cap E_{0} .
\end{aligned}
$$

Обозначим

$$
E=E_{0} \backslash\left(\bigcup_{i=1}^{j} E_{m_{i}}\right)
$$

Из (72) следует, что

$$
\mu\left(\bigcup_{i=1}^{j} E_{m_{i}}\right) \leqslant \sum_{i=1}^{j} \mu E_{m_{i}}<\sum_{i=1}^{j}\left(\varepsilon \mu \Delta_{i}^{\prime}+\frac{\varepsilon}{2 j}\right)=\varepsilon+\frac{\varepsilon}{2}=\frac{3}{2} \varepsilon .
$$


Отсюда с учетом (67) и (76) получаем, что $\mu E>1-2 \varepsilon$.

Пусть $x \in E$. Тогда для некоторого $q, 1 \leqslant q \leqslant j$, имеем $x \in \Delta_{q}^{\prime}$. Из (68), (70), $(71),(73)$ и (76) получаем, что

$$
\begin{aligned}
\left|\sum_{i=1}^{j} P_{m_{i}}(x)-\varphi(x)\right| & \leqslant\left|P_{m_{q}}(x)-\varphi(x)\right|+\sum_{\substack{i=1 \\
i \neq q}}^{j}\left|P_{m_{i}}(x)\right| \\
& \leqslant\left|P_{m_{q}}(x)-d_{q}^{\prime}\right|+\left|\varphi(x)-d_{q}^{\prime}\right|+\sum_{\substack{i=1 \\
i \neq q}}^{j} \eta_{m_{i}} \leqslant \frac{\delta}{2}+\sum_{i=1}^{j} \eta_{m_{i}}<\delta .
\end{aligned}
$$

А из (68), (70), (74) и (75) следует, что

$$
\begin{aligned}
S^{*}\left(\sum_{i=1}^{j} P_{m_{i}}, x\right) & \leqslant S^{*}\left(P_{m_{q}}, x\right)+\sum_{i \neq q} S^{*}\left(P_{m_{i}}, x\right) \\
& \leqslant C \frac{|\varphi(x)|}{\varepsilon}+2 \varepsilon+\sum_{i=1}^{j} \eta_{m_{i}}<C \frac{|\varphi(x)|}{\varepsilon}+3 \varepsilon
\end{aligned}
$$

Лемма доказана.

Пусть $f(x)$ - п.в. конечная измеримая функция, определенная на отрезке $[0,1]$, а $\left\{\gamma_{n}\right\}$ - последовательность положительных чисел, удовлетворяющая условиям

$$
\sum_{m=1}^{\infty} \gamma_{m}<+\infty, \quad 1>\gamma_{1}>\gamma_{2}>\cdots
$$

В силу леммы $5\left(\varphi(x)=f(x), \varepsilon=\gamma_{1} / 2, \delta=\gamma_{2}^{2} / 2, m=0\right)$ сушествуют полином $Q_{1}(x)=\sum_{i=1}^{j_{1}} P_{m_{i}^{\prime}}(x), 0<m_{1}^{\prime}<m_{2}^{\prime}<\cdots<m_{j_{1}}^{\prime}$, и множество $F_{1}$ такие, что

$$
\begin{aligned}
\mu F_{1} & >1-\gamma_{1}, \\
\left|f(x)-Q_{1}(x)\right| & <\frac{\gamma_{2}^{2}}{2}, \quad x \in F_{1} .
\end{aligned}
$$

Пусть уже построены полиномы $Q_{1}(x), Q_{2}(x), \ldots, Q_{k}(x)$ и множества $F_{1}, F_{2}, \ldots$ $\ldots, F_{k}$ такие, что

$$
\begin{gathered}
\mu F_{i}>1-\gamma_{i}, \quad i=1,2, \ldots, k \\
\left|f(x)-\sum_{i=1}^{k} Q_{i}(x)\right|<\frac{\gamma_{k+1}^{2}}{2}, \quad x \in F_{k} .
\end{gathered}
$$

Положим

$$
f_{k}(x)=f(x)-\sum_{i=1}^{k} Q_{i}(x) .
$$


В силу леммы $5\left(\varphi(x)=f_{k}(x), \varepsilon=\gamma_{k+1} / 2, \delta=\gamma_{k+2}^{2} / 2, m=m_{j_{k}}^{k}\right)$ существуют полином $Q_{k+1}(x)=\sum_{i=1}^{j_{k+1}} P_{m_{i}^{k+1}}(x), m_{j_{k}}^{k}<m_{1}^{k+1}<m_{2}^{k+1}<\cdots<m_{j_{k+1}}^{k+1}$, и множество $F_{k+1}$ такие, что

$$
\begin{aligned}
\mu F_{k+1} & >1-\gamma_{k+1}, \\
\left|f_{k}(x)-Q_{k+1}(x)\right| & <\frac{\gamma_{k+2}^{2}}{2}, \quad x \in F_{k+1},
\end{aligned}
$$

и

$$
S^{*}\left(Q_{k+1}, x\right)<C \frac{2\left|f_{k}(x)\right|}{\gamma_{k+1}}+\frac{\gamma_{k+1}}{2} \text { для всех } x \in F_{k+1}
$$

Из (79)-(81) следует, что на множестве $F_{k+1}^{\prime}=F_{k} \cap F_{k+1}$

$$
S^{*}\left(Q_{k+1}, x\right) \leqslant C \gamma_{k+1}+\frac{\gamma_{k+1}}{2}<(C+1) \gamma_{k+1}
$$

Так по индукции мы построим множества $F_{k}, k=1,2, \ldots$, и ряд

$$
\sum_{n=1}^{\infty} b_{n} W_{n}(x) \equiv \sum_{k=1}^{\infty} Q_{k}(x)
$$

со следуюшими свойствами:

1) ряд (83) имеет вид $\sum_{n=0}^{\infty} \delta_{n} a_{n} W_{n}(x)$, где $\delta_{n}=0, \pm 1$;

2) для любого $k \geqslant 1$ выполняются неравенства (78) и (79);

3 ) для любого $k \geqslant 1$ на множестве $F_{k+1}^{\prime}$ вьполняется неравенство (82).

Это означает, что ряд (83) на множестве

$$
F_{0} \bigcup_{m=1}^{\infty} \bigcap_{k=m}^{\infty} F_{k}^{\prime}, \quad F_{1}^{\prime}=[0,1]
$$

сходится к функции $f(x)$.

Из (77), (78) и (84) следует, что

$$
\mu F_{0}=\lim _{m \rightarrow \infty} \mu\left(\bigcap_{k=m}^{\infty} F_{k}^{\prime}\right) \geqslant \lim _{m \rightarrow \infty}\left(1-\sum_{k=m}^{\infty}\left(\gamma_{k}+\gamma_{k+1}\right)\right)=1
$$

Теорема доказана. 


\section{Список литературы}

1. Голубов Б.И., Ефимов А.Ф., Скворцов В.А. Ряды и преобразования Уолша. М.: Наука, 1987.

2. Gevorkian G. G. On coefficients of null-series and on sets of uniqueness of trigonometric and Walsh systems // Analysis Math. 1988. V. 14. P. 219-251.

3. Ульянов П. Л. Решенные и нерешенные проблемы теории тригонометрических и ортогональных рядов // УМН. 1964. Т. 19. № 1. С. 3-69.

4. Арутюнян $\Phi . Г$. Представление функций из $L_{p}, 0 \leqslant p<1$, тригонометрическими рядами с быстро убывающими коэффициентами // Изв. АН АрмССР. Сер. матем. 1984. T. 19. C. $448-466$.

5. Погосян Н.Б. О коэффициентах тригонометрических нуль-рядов // Analysis Math. 1985. V. 11. P. 139-177.

6. Körner T. W. Uniqueness for trigonometric series // Annals of Math. 1987. V. 125. P. $1-34$.

7. Навасардян K. А. О нуль-рядах по двойной системе Уолша // Изв. НАН Армении. Сер. матем. 1994. Т. 29. № 1. С. 59-78.

8. Navasardian K.A. Universal series by multiple Walsh system // J. of Contemporary Math. Analysis. V. 30. № 5. P. 14-29.

9. Меньшов Д. Е. Об универсальных тригонометрических рядах // ДАН СССР. 1945. T. 49. С. $79-82$.

10. Меньшов Д. Е. О частных суммах тригонометрических рядов // Матем. сб. 1947. T. 20(62). C. 197-237.

11. Талалян A.A. Тригонометрические ряды, универсальные относительно подрядов // Изв. АН СССР. Сер. матем. 1963. Т. 27. № 3. С. 621-660.

12. Мушезян Г. М. Об универсальных рядах относительно перестановок // Изв. АН Арм. ССР. Сер. матем. 1977. Т. 12. № 4. С. 278-302.

13. Погосян Н. Б. Представление измеримых функций ортогональными рядами // Матем. сб. 1975. Т. 98(140). С. 102-112.

14. Талалян A. А. Представление измеримых функций рядами // УМН. 1960. Т. 15. №5. C. $77-141$.

15. Талалян А. А., Овсепян Р. И. Теоремы Д. Е. Меньшова о представлении и их влияние на развитие метрической теории функций // УМН. 1992. Т. 47. № 5. С. 15-42.

16. Погосян Н. Б. Об универсальных рядах Фурье // УМН. 1983. Т. 38. № 1. С. 185-186.

17. Тиман М.., Рубинштейн А.И. О вложении классов функций, определенных на нуль-мерных группах // Изв. ВУЗов. Математика. 1980. № 8. С. 66-76.

18. Moricz F. On Walsh series with coefficients tending monotonically to zero // Acta Math. Acad. Sci. Hung. 1981. V. 38. № 1-4. P. 183-189.

19. Кашин Б. С., Саакян А. А. Ортогональные ряды. М.: Наука, 1984.

20. Качмаж C., Штейгауз Г. Теория ортогональных рядов. М.: Физматгиз, 1958.

21. Арутюнян Ф. Г. О рядах по системе Хаара // Докл. АН АрмССР. 1966. Т. 42. №3. C. $134-140$.

22. Gundy R. Martingale theory and pointwise convergence of certain orthogonal series // Trans. Amer. Math. Soc. 1966. V. 124. № 2. P. 228-248.

23. Chow Y.S. Convergence Theorems of martingales // Z. Wahrscheinlickeitstheorie und Verw. Gebiete. 1962. Bd. 1. P. 340-346. 